\title{
STRUCTURE DETERMINATION OF THE CLEAN Co(11̄̄o) SURFACE BY LEED
}

\author{
M. WELZ, W. MORITZ and D. WOLF \\ Institut für Kristallographie und Mineralogie der Universität München, SFB 128, Theresienstrasse 4I, \\ D-8000 München 2, Fed. Rep. of Germany
}

Received 27 Scptember 1982; accepted for publication 24 November 1982

\begin{abstract}
The atomic structure of the $(11 \overline{2} 0)$ surface of cobalt has been determined by LEED using six intensity spectra at normal incidence. The surface exhibits the truncated bulk structure with a contraction of the first interlayer spacing by about $8.5 \%$ with respect to the bulk value. Quantitative evaluation of the LEED spectra was done using Zanazzi and Jona's and Pendry's $r$-factors. The minimum averaged $r$-factors are $\bar{r}_{\mathrm{ZJ}}=0.09$ and $\bar{r}_{\mathrm{p}}=0.22$. No change of the interatomic distances within the plane could be detected and no rearrangement of the surface structure takes place up to temperatures shortly below the transition temperature.
\end{abstract}

\section{Introduction}

Relatively few studies dealing with single crystal cobalt surfaces have been published up to now. Of the clean surfaces only the (0001) surface of the hexagonal phase [1-3], and the (111) face [3] and (100) face [4] of the cubic phase have been investigated by a LEED analysis. The only quantitative structure determination of an adsorption system has been reported for oxygen on $\mathrm{Co}(100)$ [5]. In all these studies the difficulty of the preparation of a clean cobalt surface is mentioned. The most detailed description of the cleaning procedure is given in ref. [6]. The difficulty of the preparation is caused by the high chemical reactivity with $\mathrm{CO}$ and mainly by the fact that the hcp-fcc transition temperature at about $700 \mathrm{~K}$ should not be exceeded when a surface of the hexagonal phase is investigated. The methods used here differ in some points from those given in refs. $[3,6]$ and will therefore be described in some detail.

The structural change in the surface during the phase transition has been observed at the (0001) surface [3] with the result that the bulk stacking sequence is maintained until the transition temperature is reached. This has also been reported recently in a study on a non-basal plane, on $\operatorname{Co}(10 \overline{1} 2)$ [7], where the structural changes during the phase transition are directly visible in the diffraction picture since the diffraction spots from both phases do not 
coincide. Similar observations have been made here. During the phase transition the intensity of the LEED spots of the hexagonal phase diminishes continuously within a small temperature range and simultaneously the spots of the cubic phase occur. No indications could be found for a surface reconstruction or a behaviour of the surface layers different from that of the bulk. The results of these studies will be published in a forthcoming paper; the purpose of this paper is to report structural data of the clean $(11 \overline{2} 0)$ surface.

\section{Experimental}

Experiments were carried out in a stainless steel UHV chamber pumped by a combination of an ion pump and a $\mathrm{Ti}$ sublimation pump. Typical base pressures of less than $5 \times 10^{-11}$ mbar were routinely obtainable. The UHV chamber was equipped with a special four-grid retarding field analyser for LEED and Auger spectroscopy with a miniaturized electron gun movable in front of the grids from normal to glancing incidence [8]. Intensities were measured with a Faraday cup with an aperture of $0.5^{\circ}$, which guaranteed the measurement of integral intensities at all energies. The movement of the Faraday cup around two axes and the data collection were computer controlled. Typical run times for an $I-V$ spectrum from $50-250 \mathrm{eV}$ in steps of 1 $\mathrm{eV}$ are about 5-10 $\mathrm{min}$.

The $\operatorname{Co}(1120)$ sample was prepared from a randomly orientated single crystal of $5 \mathrm{~N}$ purity, produced by Metals Research Ltd. Using X-ray techniques, the crystal was orientated to within $0.5^{\circ}$ of the [110] direction. After cutting and grinding by spark erosion the specimen surface has been electrochemically polished in two steps: first a pretreatment in a 60:40 mixture of $\mathrm{HCl} / \mathrm{C}_{2} \mathrm{H}_{5} \mathrm{OH}$ with current densities of $1-2 \mathrm{~A} / \mathrm{cm}^{2}$, and second an additional electrochemical polishing in a $50: 20: 30$ solution of $\mathrm{H}_{3} \mathrm{PO}_{4} / \mathrm{H}_{2} \mathrm{O} / \mathrm{C}_{2} \mathrm{H}_{5} \mathrm{OH}$ with a lower current density of 10 to $50 \mathrm{~mA} / \mathrm{cm}^{2}$. After this treatment the crystal appeared smooth on an optical microscopic scale.

The prepared specimen with a final size of $9 \mathrm{~mm}$ diameter and $5 \mathrm{~mm}$ thickness was mounted on the crystal support using high purity cobalt foil. Indirect radiation heating has been used for crystal temperatures up to $1100 \mathrm{~K}$; higher temperatures were obtainable by additional electron bombardment. However, for the preparation of the clean surface of the hexagonal phase the crystal was not heated above the transition temperature of $700 \mathrm{~K}$. The temperature was measured by means of a chromel-constantan thermocouple attached to the cobalt surface.

A rather tedious cleaning procedure was necessary to get the surface suitable for LEED investigations. Auger electron spectroscopy showed the initial surface to be extensively contaminated with sulfur and carbon, and in minor concentrations also by chlorine and oxygen. Argon ion bombardment at 

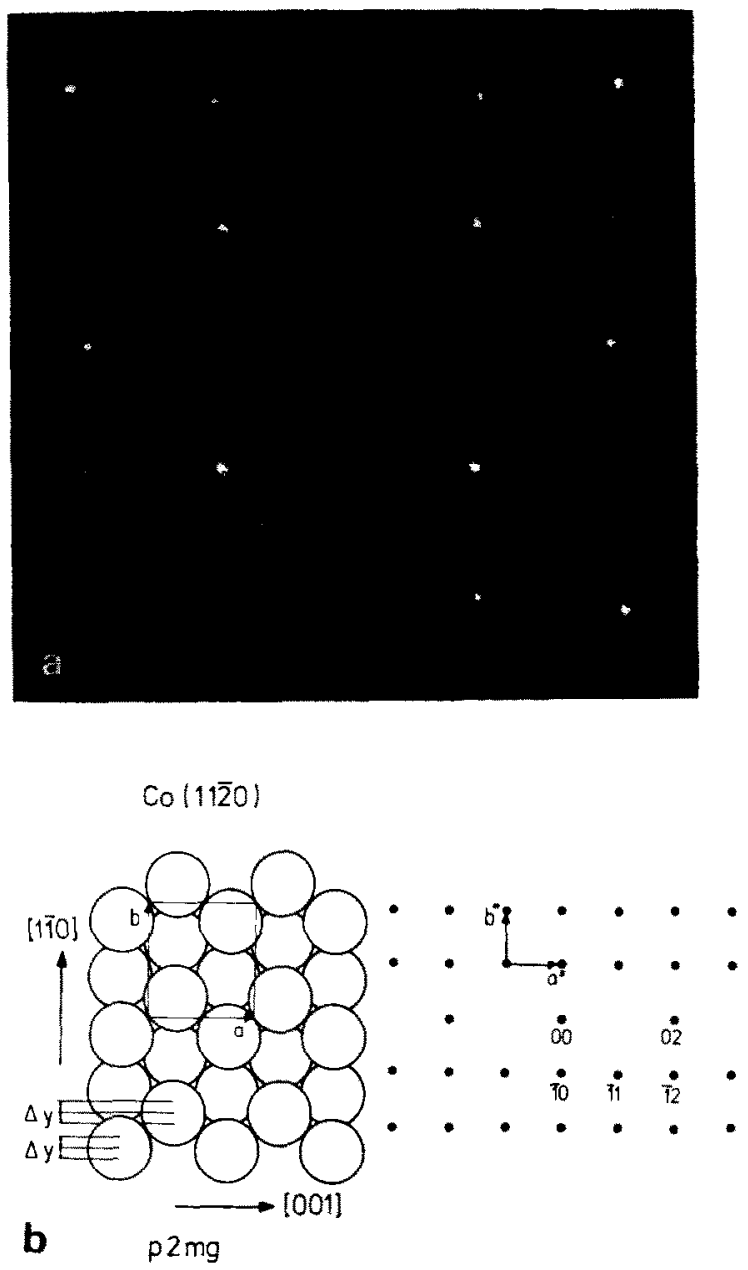

Fig. 1. (a) LEED pattern of the clean surface, $130 \mathrm{eV}$, and (b) model of the surface. $\Delta y$ indicates the shift of the atoms in the surface layer along [110] which has been considered in the calculations.

$140-300 \mathrm{eV}$ and beam currents up to $3 \mu \mathrm{A} / \mathrm{cm}^{2}$ for $0.5-2 \mathrm{~h}$ removed the contaminant layers, but the following indispensable annealing resulted in surface segregation of sulfur and carbon from the bulk. Care was taken to avoid annealing temperatures higher than $650 \mathrm{~K}$ and therefore more than 100 ion bombardment-tempering cycles were necessary until the segregation of bulk impurities to the surface was sufficiently reduced and the surface could be kept clean at $650 \mathrm{~K}$ for $24 \mathrm{~h}$. 
A further difficulty encountered during preparation was the contamination with carbon from the $\mathrm{CO}$ produced by the filament of the ion gun. This contamination could be avoided by ion bombardment with a mixture of neon and oxygen in a ratio of $1000: 1$ at a crystal temperature of $550 \mathrm{~K}$ for several minutes. The oxygen remaining at the surface after this procedure was removed with a short ion bombardment with pure neon. Neon was used at this stage of preparation to minimize sputtering damage and to avoid high annealing temperatures.

After preparation the surface exhibited a very sharp LEED pattern with low background. No streaks or superstructures were visible and the extinction of the $(0 k)$ beams with $k=2 n+1$ corresponds to the glide plane (1 $\overline{1} 0)$ of the bulk structure. The diffraction picture and the model of the surface are shown in fig. 1.

The crystal surface stayed clean for about $30 \mathrm{~min}$ at $1 \times 10^{-10} \mathrm{mbar}$ and at room temperature. At $600 \mathrm{~K}$ it took about $3 \mathrm{~h}$ before the first traces of contaminants were detectable in the Auger spectrum. The LEED intensity measurements were therefore performed at a temperature of $600 \mathrm{~K}$. Due to the relatively high Debye temperature of cobalt, the LEED intensities were not too strongly damped by the temperature factor and the $I-V$ spectra exhibited enough details necessary for structure analysis. The intensity spectra were corrected for background intensity and for the contact potential difference of about $3.5 \mathrm{eV}$ between the cobalt crystal and the electron gun cathode.

\section{Details of calculation}

Dynamical LEED calculations were performed using an atomic potential obtained from band structure calculations, which has been successfully used in the LEED analysis of the $\mathrm{Co}(0001), \mathrm{Co}(111)[2,3]$ and $\mathrm{Co}(100)$ faces [4]. Up to eight phase shifts were used, the number depending on the energy. As usual, intralayer multiple scattering was calculated in angular momentum space, which involved here two atoms per planar unit cell (see fig. 1). Multiple scattering between layers was treated by the layer doubling method since the RFS scheme turned out to be far from convergent. Even with the layer doubling scheme the scattering properties of cobalt and the low interlayer distances made it necessary to include an unusual large number of beams until convergence was reached. The criterion for cutting off the beam expansion of the wave field between the layers had to be set to $1 \times 10^{-6}$, which means that the last Fourier component is damped by this factor after propagation between two layers. This corresponds to a maximum number of 102 symmetrized beams at $250 \mathrm{eV}$. A lower number of beams did not only produce spurious peaks in the $I-V$ spectra at certain energies, but also wrong results at other energies where the layer doubling scheme showed numerically good convergence. It was 
not possible to overcome the lack of convergence by cutting off the beam expansion earlier, as proposed by Moore et al. [9], since at most energies it has been found that the intensity changed smoothly as a function of the number of beams, and only occasionally the final value was reached with a lower number of beams.

In symmetrizing the beam set, use was made of the full $\mathrm{p} 2 \mathrm{mg}$ symmetry. To increase the efficiency of the calculation and to reduce the memory space a further simplification was used in the layer doubling scheme. Two matrices, reflection and transmission, describe the scattering properties of a single layer when the layer is symmetric with respect to incidence from both sides. For a stack of layers $\mathrm{ABAB} \ldots$ this symmetry is lost, and one usually needs four matrices when a symmetrized beam set is used even when the $A$ and $B$ layers differ only in a shift of the origin and are identical otherwise. However, when the stack of layers contains a glide plane, this symmetry can be easily used. Only two matrices need to be calculated in each layer doubling step, thus saving half of the calculation time in this part of the programm. This simplification is in general possible for bulk lattice beams only, for superstructure beams still four matrices are needed.

The non-structural parameters in the calculation were $5 \mathrm{eV}$ as damping constant, the imaginary part of the inner potential, independent of energy. The Debye temperature was set to $385 \mathrm{~K}$ [10] for all layers; a variation of the surface Debye temperature has not been performed. As usual, the real part of the inner potential is found by comparison between experimental and theoretical $I-V$ curves, and is also taken to be independent of energy.

Two parameters were varied in the calculations. In addition to variation of layer spacings, a lateral shift of atomic positions along [1 $1 \overline{1} 0]$ (see fig. 1) is allowed, maintaining the symmetry. The top layer spacing has been varied from the bulk value $1.25 \AA$ to $1.07 \AA$ in steps of $0.03 \AA$ and the atoms in the top layer were moved along the $y$-axis in both directions from the bulk position by $-0.05 \AA$ to $+0.10 \AA$ in steps of $0.05 \AA$. This corresponds to a change in the bond length from 2.44 to $2.68 \AA$; the bulk equilibrium value is $2.507 \AA$.

\section{Results}

Quantitative comparison between experiment and theory was done by two $r$-factors, defined by Zanazzi and Jona [11], and by Pendry [12]. The averaged $r$-factors for six beams at normal incidence as a function of the two geometrical parameters which were varied in the calculations are shown in fig. 2 . The $r$-factor functions are plotted at several values of the inner potential, and a probably existent energy dependence of the inner potential has not been investigated. The experimental $I-V$ curves together with the theoretical ones calculated with $d_{1}=1.16 \AA$ and $\Delta y=0$ are shown in fig. 3 . 

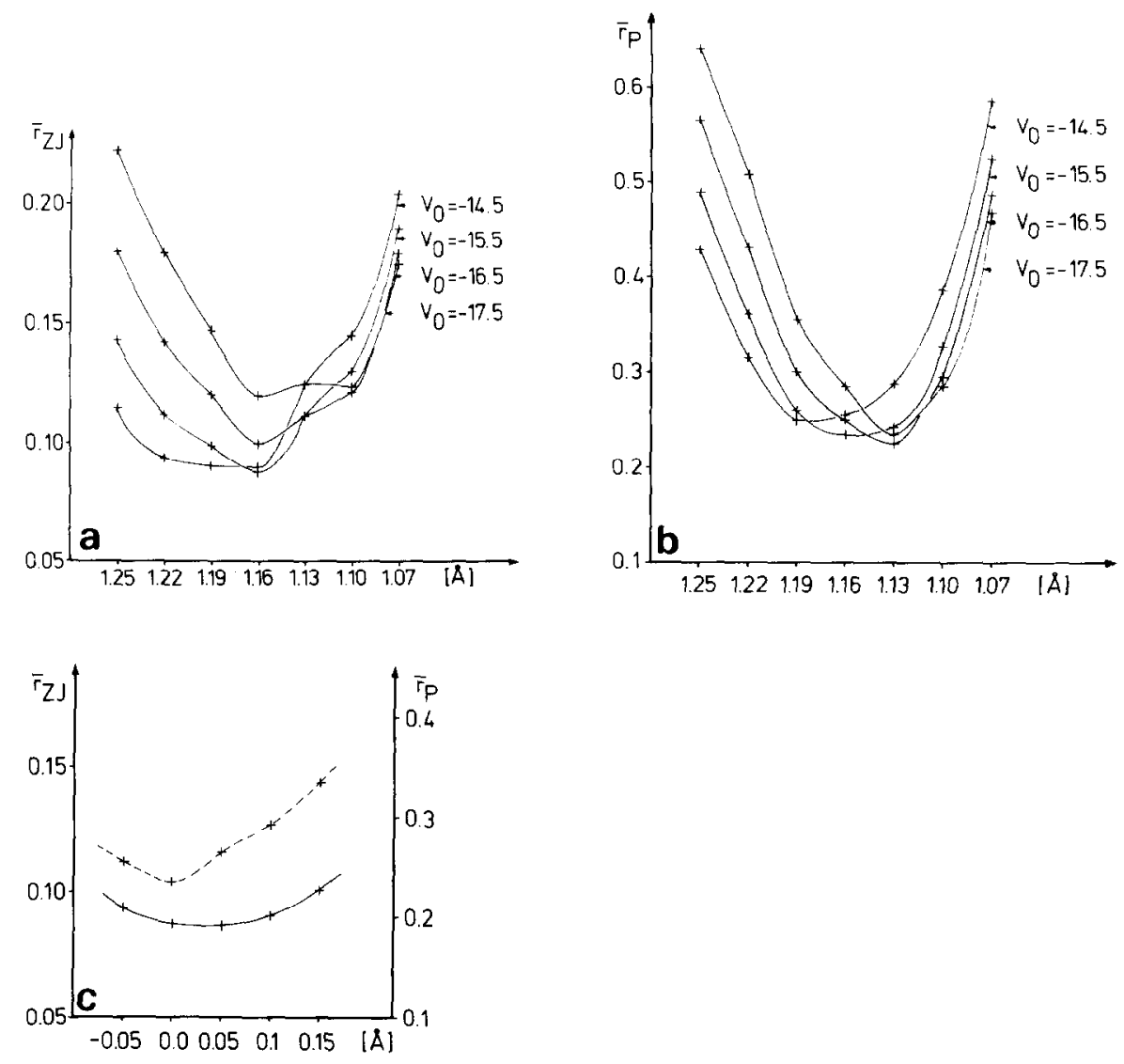

Fig. 2. Mean $r$-factors $\bar{r}_{\mathrm{P}}$ and $\bar{r}_{\mathrm{ZJ}}$ as a function of the first interlayer spacing for several values of the inner potential (a and b), and as a function of the lateral displacement $\Delta y$ in [110] of surface atoms (c). Solid line: $\bar{r}_{\mathrm{ZJ}}$, left scale; dashed line: $\bar{r}_{\mathrm{P}}$, right scale. both mean $r$-factors at the same inner potential $V_{0}=-15.5 \mathrm{eV}$, and $d_{1}=1.16 \AA$.

Both $r$-factor analyses lead to the same results within the error limit. Zanazzi and Jona's $r$-factor has a minimum value $\bar{r}_{\mathrm{ZJ}}=0.088$ at a top layer spacing $d_{1}=1.16 \AA$, corresponding to a contraction of $7.2 \%$ compared to the bulk value, $d_{\mathrm{B}}=1.25 \AA$, and at an inner potential $V_{0}=-15.5 \mathrm{eV}$. The occurence of a slight lateral shift towards an enlargement of the bond length within the plane (see fig. 1) may be concluded from the $r$-factor function $\bar{r}_{\mathrm{ZJ}}$ shown in fig. $2 c$, but it is certainly below the error limit. This $r$-factor compares the positions of the maxima and minima and also the shape of the curves via the second derivatives, while Pendry's $r$-factor emphasizes the positions of the 

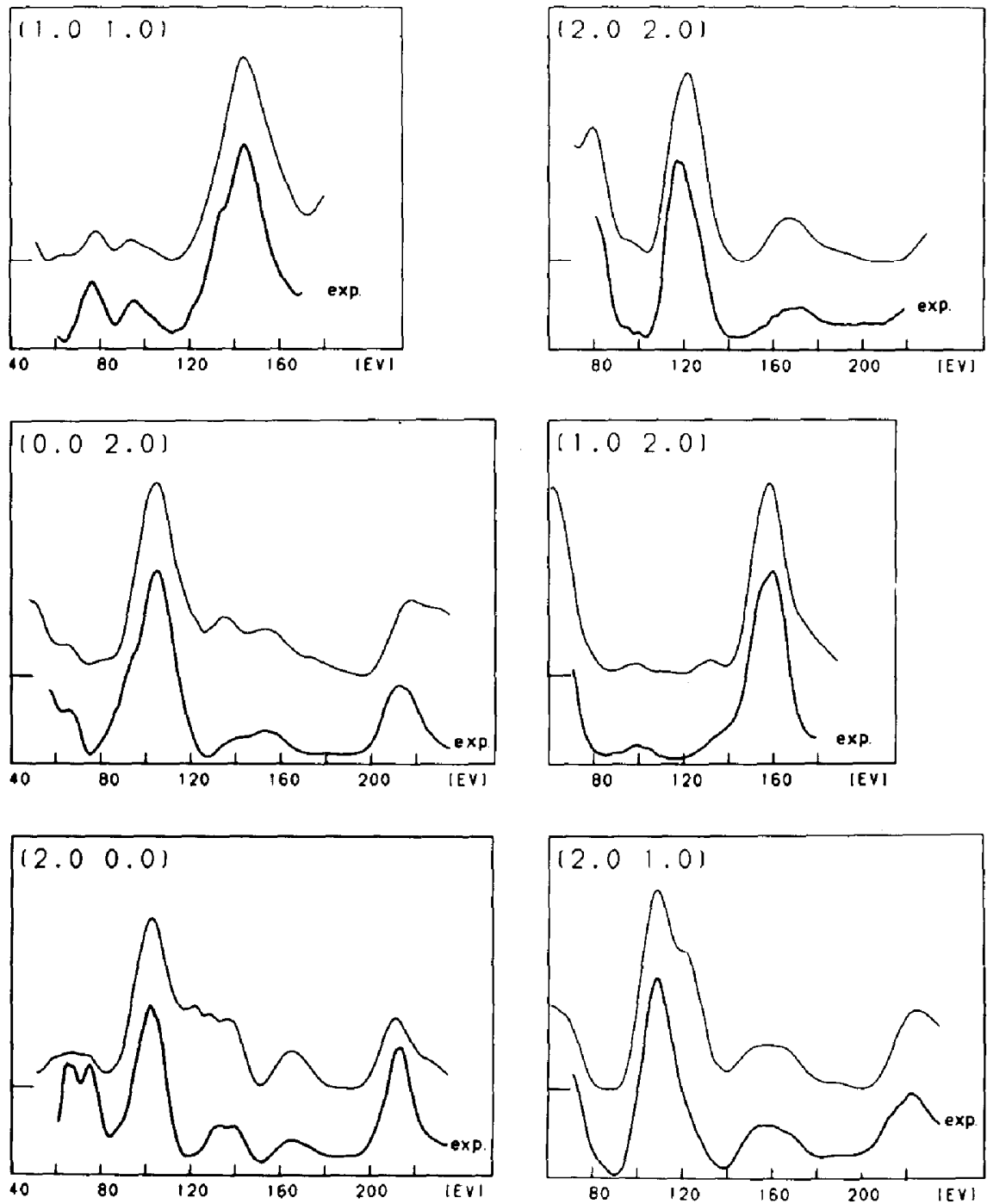

Fig. 3. Experimental and theoretical $I-V$ spectra at normal incidence, $T=600 \mathrm{~K}, V_{0}=-15.5 \mathrm{eV}$.

peaks. The results are therefore slightly different. Pendry's $r$-factor leads to a top layer spacing $d_{1}=1.13 \AA$ and $9.6 \%$ contraction, and no indications are found for a lateral shift in the top layer. We finally conclude that a contraction by $8.5 \pm 3 \%$ of the first layer spacing exists, and that no change in the bond lengths parallel to the surface occurs. The error of $3 \%$ is estimated from the spread of the minima of the $r$-factors of the single beams and from the 
consideration that the real part of the inner potential is energy dependent as discussed below. On the close packed surfaces of the hexagonal and the cubic phase no contraction was found [2,3], and on the cubic (100) face about 4\% [4]. The higher contraction found here is in agreement with the observations made on other materials that the close packed surfaces exhibit little or no contraction of the top layer spacing and the contraction increases the more open is the surface.

The values for the inner potential found by shifting the theoretical curves in the $r$-factor analysis are $-15.5 \mathrm{eV}$ for Zanazzi and Jona's $r$-factor and -16.5 $\mathrm{eV}$ for Pendry's $r$-factor. Both values are within the error limits of this analysis and also compare well to the values found at other clean cobalt surfaces, namely $-16 \mathrm{eV}$ at $\mathrm{Co}(0001)$ [3] and $-16.5 \mathrm{eV}$ at $\mathrm{Co}(100)$ [4]. The inner potential certainly depends on the energy of the incident beam and may change by several eV within the energy range considered here [13]. This uncertainty causes a spread of the minima of the single $r$-factor curves and a broad minimum in the mean $r$-factor curve. Besides other ambiguities in the way of calculating the potential, this effect limits the accuracy of the structure analysis. The precision for the atomic distances cannot be greater than that for the wave length with which they are measured. Taking a constant average value for the inner potential means an error of not more than $1 \%$ in the wave length at the lowest and highest energy. At the backscattering geometry used by LEED this leads to an error of about $2 \%$ in the structure parameters.

\section{Acknowledgements}

We thank Prof. Dr. H. Jagodzinski for helpful discussions and Dr. M.A. Van Hove for submitting the Co potential. Financial support from the Deutsche Forschungsgemeinschaft SFB 128 is gratefully acknowledged.

\section{References}

[1] G.L.P. Berning, Surface Sci. 61 (1976) 673.

[2] G.L.P. Berning, G.P. Alldridge and P.E. Viljoen, Surface Sci. 104 (1981) L225.

[3] B.W. Lee, R. Alsenz, A. Ignatiev and M.A. Van Hove, Phys. Rev. 17 (1978) 1510.

[4] M. Maglietta, E. Zanazzi, F. Jona, D.W. Jepsen and P.M. Marcus, Appl. Phys. 15 (1978) 409.

[5] M. Maglietta, E. Zanazzi, U. Bardi, F. Jona, D.W. Jepsen and P.M. Marcus, Surface Sci. 77 (1978) 101.

[6] M. Maglietta and G. Rovida, Surface Sci. 71 (1978) 495.

[7] K.A. Prior, K. Schwaha and R.M. Lambert, Surface Sci. 77 (1978) 193.

[8] D. Wolf, H. Jagodzinski and W. Moritz, Surface Sci. 77 (1978) 265.

[9] W.T. Moore, S.J. White, D.C. Frost and K.A.R. Mitchell, Surface Sci. 116 (1982) 253.

[10] International Tables, Vol. 3 (Kynoch, Birmingham, 1962).

[11] E. Zanazzi and F. Jona, Surface Sci. 62 (1977) 61.

[12] J.B. Pendry, J. Phys. C13 (1980) 937.

[13] J.E. Demuth, P.M. Marcus and D.W. Jepsen, Phys. Rev. B11 (1975) 1460. 